\title{
Femtosecond X-ray Pulses from a Synchrotron
}

\author{
R.W. Schoenlein ${ }^{1}$, H.H.W. Chong ${ }^{2}$, T.E. Glover ${ }^{3}$, P.A. Heimann ${ }^{3}$, C.V. Shank ${ }^{1}$, \\ A.A. Zholents ${ }^{4}$, and M.S. Zolotorev ${ }^{4}$ \\ ${ }^{1}$ Materials Sciences Division, Ernest Orlando Lawrence Berkeley National Laboratory, \\ 1 Cyclotron Rd. MS: 2-300, Berkeley CA 94720, USA. \\ E-mail: rwschoenlein@lbl.gov \\ 2 Applied Science and Technology Graduate Group, University of California Berkeley, \\ Berkeley CA 94720, USA. \\ ${ }^{3}$ Advanced Light Source Division, Lawrence Berkeley National Laboratory \\ ${ }^{4}$ Accelerator and Fusion Research Division, Lawrence Berkeley National Laboratory
}

\begin{abstract}
An important frontier in ultrafast science is the application of femtosecond x-ray pulses to the study of structural dynamics in condensed matter. We show that femtosecond laser pulses can be used to generate high-brightness, tunable, femtosecond x-ray pulses from a synchrotron. Performance of existing and proposed femtosecond $\mathrm{x}$-ray beamlines at the Advanced Light Source synchrotron are discussed.
\end{abstract}

\section{Introduction}

An important new area of scientific research in chemistry, physics, and biology is the investigation of ultrafast structural dynamics and atomic motion in condensed matter using femtosecond x-ray pulses. X-rays are powerful probes of atomic structure since they interact with core electronic levels, and can therefore provide direct information about relative atomic positions and coordination. Synchrotrons generating high flux (photons $/ \mathrm{sec} / 0.1 \% \quad \mathrm{BW}$ ), and high-brightness (photons $/ \mathrm{sec} / \mathrm{mm}^{2} / \mathrm{mrad}^{2} / 0.1 \% \mathrm{BW}$ ) x-ray beams have proven to be powerful tools for probing the "static" structure of matter. Techniques such as x-ray diffraction, extended x-ray absorption fine structure (EXAFS) and many others are widely used at such facilities to obtain structural information with a resolution of much less than $1 \AA$. However, the time resolution provided by synchrotrons is limited to $>30 \mathrm{ps}$, as determined by the length of the stored electron bunches. This is orders of magnitude longer than the fundamental time scale for atomic motion, dictated by the period of an atomic vibration, $\sim 100 \mathrm{fs}$. We demonstrate a novel scheme for generating ultrashort pulses of synchrotron radiation[1]. Femtosecond synchrotron pulses are directly measured from a bend-magnet beamline at the Advanced Light Source (ALS)[2, 3]. A femtosecond x-ray beamline based on a bend-magnet is presently being commissioned at the ALS, and a femtosecond undulator beamline providing higher flux and brightness is proposed.

\section{Experimental Methods}

The time structure of synchrotron radiation is determined by the duration of the stored electron bunches, typically longer than $30 \mathrm{ps}$. Storage of ultrashort relativistic electron bunches is problematic due to instabilities arising from bunch- 
induced wakefields and coherent synchrotron radiation. However, femtosecondduration electron bunches can exist in a storage ring for a limited time. Our approach is to create femtosecond time-structure on a long electron bunch by using a femtosecond laser pulse to modulate the energy of an ultrashort slice of the bunch.
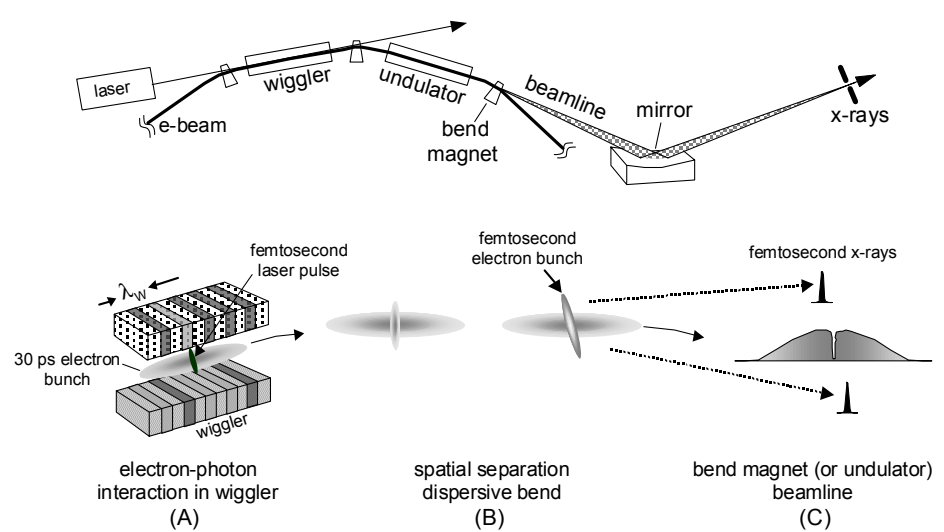

Fig. 1. Schematic of method for generating femtosecond synchrotron pulses. (A) Femtosecond laser pulse interaction with the electron bunch in a resonantly-tuned wiggler. (B) Transverse separation of modulated electrons in a dispersive bend of the storage ring. (C) Generation of synchrotron radiation from a bend-magnet (or undulator) and separation of the femtosecond synchrotron radiation at the beamline image plane.

Figure 1 illustrates the modulation and generation scheme. A femtosecond laser pulse co-propagates with the stored electron bunch through a wiggler (Fig. 1A). The high electric field of the laser pulse $\left(\sim 10^{9} \mathrm{~V} / \mathrm{m}\right)$ modulates the energy of the underlying electrons as they traverse the wiggler (electrons are accelerated or decelerated depending on the optical phase, $\phi$, seen by each electron at the entrance of the wiggler). The optimal interaction occurs when the central wavelength of the spontaneous emission from an electron passing through the wiggler, $\lambda_{S}=\lambda_{W}\left(1+K^{2} / 2\right) / 2 \gamma^{2}$, satisfies the resonance condition $\lambda_{S}=\lambda_{L}$ where $\lambda_{L}$ is the laser wavelength, $\lambda_{\mathrm{W}}$ is the wiggler period, $\gamma$ is the Lorentz factor, and the deflection parameter $\mathrm{K}=\mathrm{eB}_{\mathrm{o}} \lambda_{\mathrm{W}} / 2 \pi \mathrm{mc}$ (where $\mathrm{B}_{\mathrm{o}}$ is the peak magnetic field of the wiggler). In addition, the transverse mode of the laser beam must match the transverse mode of the spontaneous radiation from the electron passing through the wiggler, and the laser spectral bandwidth must match the spectrum of the fundamental wiggler radiation averaged over the transverse mode. Under these conditions, the electron energy modulation, $\Delta \mathrm{E}$, is given by

$$
\Delta E=2\left(A_{L} A_{W} \frac{M_{W}}{\sqrt{2} M_{L}} \eta_{e m i t}\right)^{1 / 2} \cos \phi
$$

where $A_{L}$ is the laser pulse energy, $M_{W}$ is the number of wiggler periods, $M_{L}$ is the laser pulse length in optical cycles (FWHM), $A_{W} \cong 4.1 \alpha h \mathrm{c} / \lambda_{\mathrm{L}} \mathrm{K}^{2} /\left(2+\mathrm{K}^{2}\right)$ is the energy spontaneously radiated by a single electron passing through the wiggler, 
and $\eta_{\text {emit }} \approx 0.7$ accounts for the non-zero electron beam size. For example: a $25 \mathrm{fs}$ laser pulse with a photon energy of $1.55 \mathrm{eV}$, and a pulse energy $A_{L}=100 \mu \mathrm{J}$ will produce an energy modulation amplitude $\Delta \mathrm{E} \approx 9 \mathrm{MeV}$ using a wiggler of 19 periods.

By creating an energy modulation that is significantly larger than the beam energy spread, the transverse dispersion of the storage ring will cause a spatial displacement of the modulated electrons from the rest of the electron bunch (Fig. 1B). Finally, by imaging the synchrotron radiation from the displaced electrons to the experimental area, femtosecond x-rays can be separated from the long-pulse using an aperture (Fig. 1C). The duration of the synchrotron radiation produced by the laser-modulated electrons will be approximately the same as the laser pulse duration. Furthermore, the extraction of an ultrashort slice of electrons leaves behind an ultrashort hole or "dark pulse" in the core of the electron bunch. This dark pulse will appear in the generated x-rays, and can also be used for timeresolved spectroscopy.

Our experiments are conducted at the ALS storage ring operating at $\mathrm{E}=1.5 \mathrm{GeV}$ (rms beam energy spread $\sigma_{\mathrm{E}}=1.2 \mathrm{MeV}$ ) using a wiggler with $\mathrm{M}_{\mathrm{W}}=19$ periods, $\lambda_{\mathrm{W}}=16 \mathrm{~cm}$, with the gap adjusted for $\mathrm{K} \cong 13$. Femtosecond pulses $\left(\tau_{\mathrm{L}}=100 \mathrm{fs}, \mathrm{A}_{\mathrm{L}}=400 \mu \mathrm{J}, \lambda_{\mathrm{L}}=800 \mathrm{~nm}, f_{\mathrm{L}}=1 \mathrm{kHz}\right.$,) from a Ti:sapphire laser system are synchronized to the storage ring RF using phase-locking techniques, and are directed into the main vacuum chamber and co-propagate with the electron beam through the wiggler. Following the interaction region, a mirror directs the fundamental spontaneous wiggler emission and the laser beam out of the storage ring to enable direct measurements of the temporal and spectral overlap, and the spatial mode matching between the laser pulses and the wiggler radiation. The efficiency of the interaction between the laser and electron beams is monitored by measuring the gain in the laser pulse energy. This gain $\left(\sim 10^{-3}\right)$ is equivalent to the single-pass gain of a free-electron laser, and is optimum under the same modematching conditions described above[3].

\section{Results and Discussion}

The time structure of the temporally incoherent synchrotron radiation is directly determined by the time structure of the electron bunch and is invariant over the entire spectrum of the synchrotron emission, from infrared to x-ray wavelengths. We directly measure the time-structure of the visible synchrotron pulses (at $\sim 2 \mathrm{eV}$ ) via cross-correlation (in BBO) with a delayed 50 fs pulse from the laser system. An adjustable knife-edge located in the beamline at an intermediate image plane provides a means to select synchrotron radiation originating from different transverse regions of the electron beam.

Figure 2 shows two cross-correlation measurements made at various knifeedge positions, in units of the rms horizontal beam size, $\sigma_{\mathrm{x}}$. Measurements of the central core of the synchrotron beam (Fig. 2A) reveal the femtosecond hole or dark pulse that is created due to the acceleration (and deceleration) of electrons by the laser pulse, and their subsequent transverse spatial separation from the central core of the bunch. The bright pulse is measured with the knife-edge at the $3 \sigma_{x}$ position (Fig. 2B) such that the central core of the beam is blocked. The solid lines in Fig. 2 are predicted pulse shapes based on a model calculation of the electron bunch 
distribution (using known parameters of the electron beam and the storage-ring lattice) [3].
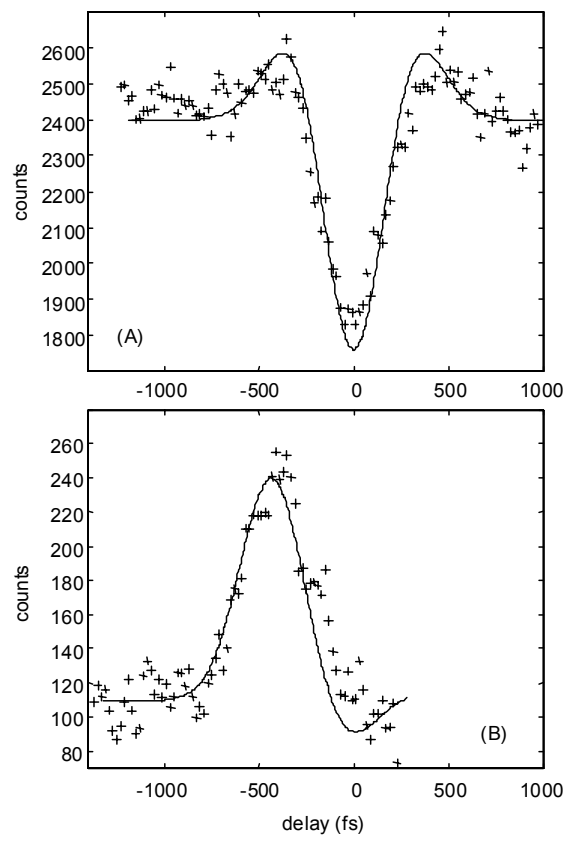

Fig. 2. Cross-correlation measurements between a delayed laser pulse and synchrotron radiation originating from an energy-modulated electron bunch. In (A), synchrotron radiation from the central core $\left( \pm 3 \sigma_{x}\right)$ of the electron bunch is selected. In (B), synchrotron radiation from the horizontal wings $\left(+3 \sigma_{\mathrm{x}}\right.$ to $\left.+8 \sigma_{\mathrm{x}}\right)$ of the electron bunch is selected. Solid lines are from a model calculation of the spatial and temporal distribution of the energymodulated electron bunch following propagation through 1.5 arc-sectors at the ALS[3].

The location of the bend-magnet beamline used in these demonstration experiments is less than optimum due to the distance from the wiggler (in which the laser/electron interaction occurs) to the radiating bend magnet. Stretching of the laser-modulated electron bunch along this storage-ring arc accounts for the observed $\sim 300$ fs pulse duration. For a bend-magnet located immediately following the wiggler, the stretching of the electron bunch will be reduced by a factor of three, resulting in an $\mathrm{x}$-ray pulse duration of $\sim 100 \mathrm{fs}$.

The average flux and brightness of the femtosecond x-rays can be determined from the full flux and brightness from an ALS bend-magnet (or undulator) beamline[4] scaled by three factors: $\eta_{1}=\tau_{\mathrm{L}} / \tau_{\mathrm{e}}, \eta_{2}=f_{\mathrm{L}} / f_{\mathrm{B}}$, and $\eta_{3} \approx 0.2$ where $\tau_{\mathrm{L}}$ and $\tau_{\mathrm{e}}$ are the laser pulse and electron bunch durations, $f_{\mathrm{L}}$ and $/ f_{\mathrm{B}}$ are the laser and electron-bunch repetition rates, and $\eta_{3}$ accounts for the fraction of electrons that are in the proper phase of the laser pulse to get the maximum energy exchange suitable for creating the large transverse separation. Synchrotron radiation damping provides for recovery of the electron beam between interactions. With a typical fill pattern of $\sim 300$ bunches in the storage ring, femtosecond $\mathrm{x}$-rays can be generated at repetition rates as high as $100 \mathrm{kHz}$ without adversely affecting the other beamlines at the ALS. 


\section{Conclusions}

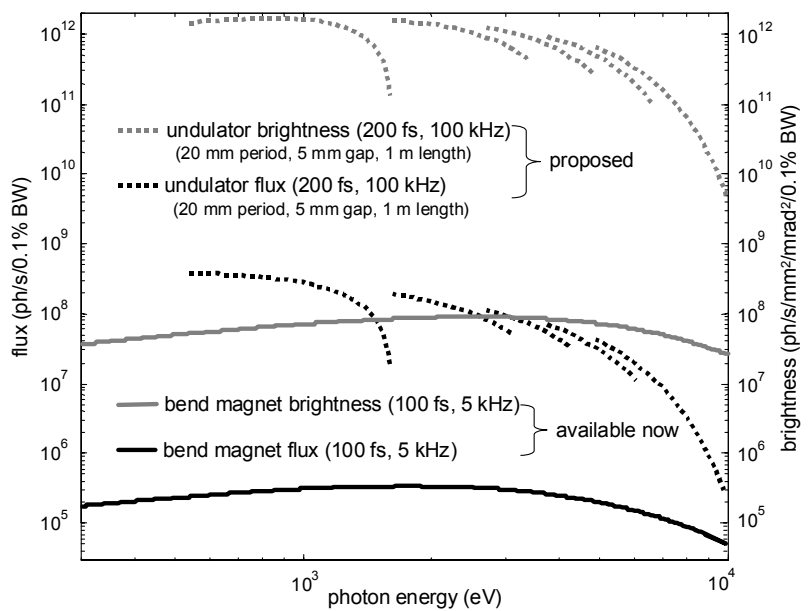

Fig. 3. Flux and brightness for two femtosecond $x$-ray beamlines based on a bend-magnet and on an undulator. The undulator spectra is the locus of narrow spectral peaks (tuned by adjusting the undulator gap) and represents the envelope of harmonics 1, 3, 5, 7, and 9.

Figure 3 shows the calculated flux and brightness from two different femtosecond x-ray beamlines assuming a beam energy of $1.9 \mathrm{GeV}$, (400 mA average current, $30 \mathrm{ps}$ bunch duration, $500 \mathrm{MHz}$ repetition rate), and rms source size of $200 \mu \mathrm{m}(\mathrm{H}) \times 20 \mu \mathrm{m}(\mathrm{V})$ in the straight sections, and $100 \mu \mathrm{m}(\mathrm{H}) \times 9 \mu \mathrm{m}$ $(\mathrm{V})$ in the bend sections. The first beamline (presently being commissioned at the ALS) is based on a bend magnet with a field of $1.27 \mathrm{~T}$ and an x-ray optic collecting $3 \mathrm{mrad}(\mathrm{H}) \times 0.3 \mathrm{mrad}(\mathrm{V})$ of the broadband bend-magnet emission. The second beamline (proposed for ALS straight sector 6) is based on a small-gap undulator with $50 \times 2 \mathrm{~cm}$ periods and a maximum deflection parameter of 2.02 ( $\sim 5 \mathrm{~mm}$ min. gap). Undulators offer substantially higher $\mathrm{x}$-ray brightness and flux (per fractional bandwidth) than bend-magnets. These two beamlines are optimally located (immediately following the wiggler) and will generate $100 \mathrm{fs}$ and $200 \mathrm{fs} \mathrm{x}$ ray pulses respectively.

Acknowledgement. Supported by the Director, Office of Science, Office of Basic Energy Sciences, of the U.S. Department of Energy under Contract No. DE-AC03-76SF00098.

\section{References}

1 A.A. Zholents, M.S. Zolotorev, Phys. Rev. Lett. 76, 912, 1996.

2 R.W. Schoenlein, S. Chattopadhyay, H.H.W. Chong, T.E. Glover, P.A. Heimann, C.V. Shank, A. Zholents, and M. Zolotorev, Science, 287, 2237, 2000.

3 R.W. Schoenlein, S. Chattopadhyay, H.H.W. Chong, T.E. Glover, P.A. Heimann, C.V. Shank, A. Zholents, and M. Zolotorev, Appl. Phys. B, (in press), 2000.

4 "1-2 GeV Synchrotron Radiation Source - Conceptual Design Report," Lawrence Berkeley Laboratory LBL PUB-5172 Rev., July 1986. 